\title{
Magnetic field errors tolerances of Nuclotron booster
}

\author{
Andrey Butenko, Olha Kazinova*, Sergey Kostromin, Vladimir Mikhaylov, Alexey Tuzikov, Hamlet Khodzhibagiyan \\ Joint Institute for Nuclear Research, Dubna, Russia
}

\begin{abstract}
Generation of magnetic field in units of booster synchrotron for the NICA project is one of the most important conditions for getting the required parameters and qualitative accelerator operation. Research of linear and nonlinear dynamics of ion beam ${ }_{197} \mathrm{Au}^{31+}$ in the booster have carried out with MADX program. Analytical estimation of magnetic field errors tolerance and numerical computation of dynamic aperture of booster DFO-magnetic lattice are presented. Closed orbit distortion with random errors of magnetic fields and errors in layout of booster units was evaluated.
\end{abstract}

\section{Magnetic lattice of booster}

The main task of this article is to evaluate maximum orbit distortion and dynamic aperture according to requirements specification for booster magnetic measurements and to find out magnetic field errors tolerances. For start let's have a look on the booster lattice. Magnetic lattice of the booster consists of 4 quadrants. Each quadrant includes 6 DFOperiods, 5 periods contain 2 dipole sector magnets and 1 period contains rectilinear gap. The booster will accelerate ${ }_{197} \mathrm{Au}^{31+}$ ions to $578 \mathrm{MeV} / \mathrm{n}$. The perimeter of the accelerating ring is $210.96 \mathrm{~m}$.

Simulation of beam motion was carried out with MAD-X program [1]. Dynamic functions in one quadrant of booster are shown in Fig.1. There are two types of errors of magnetic field:

1. nonlinearity of third and higher orders - systematic errors, existing in every unit and determining magnet design;

2. random errors, appearing because of magnet production inaccuracy and inaccuracy of placement along equilibrium closed orbit of the accelerator.

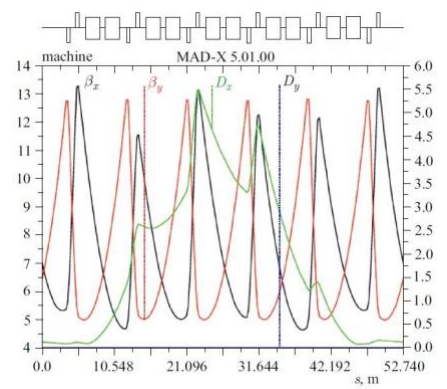

Fig. 1. $\beta_{x, y}$ and dispersion in one superperiod of magnetic lattice.

\section{Analytical estimation of magnetic field errors tolerance}

The main orbit distorters are:

1. effective length spread $\mathrm{L}_{\text {eff }}$ and inductions $<\Delta \mathrm{B}_{\mathrm{Z}} / \mathrm{B}>_{\mathrm{o}}$, $\mathrm{m}$ in dipole magnets;

2. magnets rotation of median plane by $<\alpha_{s}>_{m}$;

3. transverse displacement of longitudinal magnetic axis for quadrupole lenses by $\langle\Delta x\rangle_{1}$ and $\langle\Delta y\rangle_{1}$.

Maximum deviation of closed orbit was estimated with harmonic method [2]. Four harmonics with numbers $m=3$, 4,5, 6 are the closest harmonics to betatron oscillation tunes. Mean square amplitudes of m-harmonic for Fourier orbit expansion determine as:

$$
\left\langle\mathrm{Y}_{m}\right\rangle=\frac{Q_{y} \beta^{1 / 2}{ }_{y, \max }}{\left|Q_{y}{ }^{2}-m^{2}\right| \pi \rho}\left[\sum_{j} m_{j}\langle\Delta B / B\rangle_{o, j}^{2} L_{J} \int_{0}^{s_{f}} \beta_{y} d s\right]^{1 / 2}
$$

where $j$ - type of unit (magnet or lens), $L_{J}$ - effective length, $m_{j}$ - number of units of the same type, $\rho$ - radius of curvature for dipole magnets. Maximum orbit deviation in accordance with procedure [2] with 98\% probability doesn't exceed the value:

$$
\mathrm{Y}_{\max }=2\left(\sum_{m=3}^{6}\left\langle\mathrm{Y}_{m}\right\rangle^{2}\right)^{1 / 2}\left(1+|\delta|-|\delta|^{2}\right)
$$

\footnotetext{
*.Kazinova: kazinova.olga@gmail.com
} 
where $|\delta|=\left|Q_{y}-m\right|, m$ - harmonic number of the closest integer resonance disturbance.

For almost symmetrical magnetic lattice of booster on gradients the coefficients in (1) and (2) for x-and y-planes are approximately equal, therefore the below estimation covers horizontal and vertical orbit projection. For $|\delta|=0.1$ mean square amplitudes of 3-6 harmonics connect with field and adjustment errors by next ratios:

$$
\begin{gathered}
\left\langle Y_{3}\right\rangle^{2}=1.4\langle\Delta B / B\rangle_{O, \mathrm{M}}^{2}+12.6\langle\Delta Y\rangle_{L}^{2} \\
\left\langle Y_{4}\right\rangle^{2}=5.2\langle\Delta B / B\rangle_{O, \mathrm{M}}^{2}+43.7\langle\Delta Y\rangle_{L}^{2} \\
\left\langle Y_{5}\right\rangle^{2}=134.8\langle\Delta B / B\rangle_{O, \mathrm{M}}^{2}+1156.2\langle\Delta Y\rangle_{L}^{2} \\
\left\langle Y_{6}\right\rangle^{2}=1.9\langle\Delta B / B\rangle_{O, \mathrm{M}}^{2}+14.8\langle\Delta Y\rangle_{L}^{2}
\end{gathered}
$$

where linear displacement $\langle\Delta \mathrm{Y}\rangle_{\mathrm{L}}$ and amplitudes $\left\langle\mathrm{Y}_{\mathrm{m}}\right\rangle$ are given in meters. Tolerances for magnetic field spread induction in dipole magnets, adjustment errors and possible displacements of units using eq.(1) are determined from inequality:

$$
632.1<\Delta B / B>{ }^{2}{ }_{O, M}+5338.5<\Delta Y>^{2}{ }_{L}<Y^{2} \max
$$

If magnetic induction spread $\left\langle\Delta B / B>_{O, M}=5 \cdot 10^{-4}\right.$, rotation angle of longitudinal axis for dipole magnets $\left\langle\alpha_{\mathrm{s}}>_{\mathrm{m}}=5 \cdot 10^{-4}\right.$ and transverse displacement of quadrupole magnets $\langle\Delta \mathrm{x}\rangle_{1}=\langle\Delta \mathrm{y}\rangle_{1}=0.1 \mathrm{~mm}$, maximum deviation of horizontal and vertical orbit projection is approximately $10 \mathrm{~mm}$. As can be seen from these inequalities, main contribution to the orbit distortion adds by $\left\langle\mathrm{Y}_{5}\right\rangle$. The percentage of its impact is $80 \%$. According to the technical project of the NICA accelerator complex [3], maximum distortion for the uncorrected orbit is $15 \mathrm{~mm}$. So, there is no exceeding the limits.

The effect of systematic and random errors on betatron tune characteristics were analyzed by standard method $[4,5]$ with help of shortened equations:

$$
\begin{gathered}
I_{x}^{\prime}=n_{x}\left|H_{r}\right| I_{x}^{\frac{n_{x} \mid}{2}} I_{y}^{\frac{n_{y} \mid}{2}} \sin \varphi \\
I_{y}^{\prime}=n_{y}\left|H_{r}\right| I_{x}^{\frac{n_{x} \mid}{2}} I_{y}^{\frac{n_{y}}{2}} \sin \varphi \\
\Phi_{x}^{\prime}=\sum_{n=1}^{10} \sum_{j-1}^{n_{0}} \sum_{k=0}^{j-1}(j-k) H_{n, j, k} I_{x}^{j-k-1} I_{y}^{k}+\left|n_{x}\right|\left|H_{r}\right| I_{x}^{\frac{\left|n_{x}\right|-2}{2}} I_{y}^{\frac{\mid n_{y}}{2}} \cos \varphi \\
\Phi_{y}^{\prime}=\sum_{n=1}^{10} \sum_{j=1}^{n_{0}} \sum_{k=1}^{j-1} k H_{n, j, k} I_{x}^{j-k} I_{y}^{k-1}+\left|n_{x}\right|\left|H_{r}\right| I_{x}^{\frac{\left|n_{x}\right|}{2}} I_{y}^{\frac{\left|n_{y}\right|}{2}} \cos \varphi
\end{gathered}
$$

where " '" - azimuth $\theta$ differentiation, $I_{x, y}(\theta)$ and $\Phi_{x, y}(\theta)$ - slowly varying function: square amplitudes and horisontal and vertical betatron tune phases,

$$
H_{n, j, k}=\frac{(-1)^{k} n ! R}{k !(j-k) !(n+1-2 j) ! \pi \rho r^{n} 2^{j+1}}\left(\frac{\Delta p}{p}\right)^{n+1-2 j} \int_{0}^{2 \pi} \beta_{x}^{j-k} \beta_{y}^{k} \psi^{n+1-2 j}\left(\frac{\Delta B_{y}}{B}\right)_{n} d \theta
$$

- coefficients which determine frequency shift and spread relatively to the selected position of operation point, $n_{0}$ integral part of $(n+1) / 2$. Parameters which define impact on the beam from one of resonances $n_{x} Q_{x}+n_{y} Q_{y}=m$ are described in (6) by the following expression:

$$
\begin{gathered}
\left|H_{r}\right|=R h_{r} \int_{0}^{2 \pi} \beta_{x}^{\frac{\left|n_{x}\right|}{2}} \beta_{y}^{\frac{\left|n_{y}\right|}{2}} e^{i\left(n_{x} \gamma_{x}+n_{y} \gamma_{y}-m \theta\right)}\left(\frac{\Delta B}{B}\right)_{n_{r}-1} d \theta \\
\varphi=n_{x} \Phi_{x}+n_{y} \Phi_{y}+\Phi_{r}+\delta \theta
\end{gathered}
$$

where $\quad h_{r}=\frac{\left(n_{r}-1\right) !}{2^{n_{r} / 2} \pi \rho r^{n_{r}-1}\left|n_{x}\right| !\left|n_{y}\right| !}, \delta=n_{x} Q_{x}+n_{y} Q_{y}-m^{-}$resonance detuning, $n_{r}=\left|n_{x}\right|+\left|n_{y}\right|$ - resonance order, $\gamma_{x, y}=\mu_{x, y}-Q_{x, y} \theta$ - argument of Floquet function, $\Phi_{r}$ - phase perturbation.

Tolerance estimation for systematic errors of magnetic field was carried out in no-resonance effect on the beam assumption. Then the values of $\Phi_{\mathrm{x}, \mathrm{y}}$ are frequency shifts and in general case depend on amplitudes and particle momentum (with constancy of betatron tune amplitude). The estimated fractional increase of emittance $\Delta \varepsilon_{x, y} / \varepsilon_{x, y}$ on the basis of detuning modulus $|\delta|$ is much bigger than the resonance band half-width. Therefore $\Phi_{x, y}(\theta)$ dependence can be neglected and the equations for square of amplitude will be:

$$
\Delta I_{x, y}=n_{x, y}\left|H_{r}\right| I_{x}^{\frac{\left|n_{x}\right|}{2}} I_{y}^{\frac{\left|n_{y}\right|}{2}} \sin \varphi \Delta \theta
$$

Whereas mean value $\sin \varphi$ equals $2 / \pi$ in the range $0<\varphi<\pi / 2$ and assuming $\Delta Q=\pi /(2|\delta|)$, we will get

$$
\frac{\Delta \varepsilon_{x, y}}{\varepsilon_{x, y}}=\frac{\left|\Delta I_{x, y}\right|}{I_{x, y}}=\frac{\left|n_{x, y}\right|}{|\delta|}\left|H_{r}\right| I_{x, y}^{\frac{\left|n_{x, y}\right|-2}{2}} I_{y, x}^{\frac{\left|n_{y, x}\right|}{2}}
$$

where $\left\langle H_{r}\right\rangle$ for resonances exciting by random errors of magnetic field doesn’t exceed with $98 \%$ probability 


$$
\left\langle H_{r}\right\rangle=h_{r}\left[\sum_{j} m_{j}\left\langle\frac{\Delta B}{B}\right\rangle^{2} S_{j} \int_{0}^{S_{j}} \beta_{x}^{\left|n_{x}\right|} \beta_{y}^{\left|n_{y}\right|} d s\right]^{1 / 2}
$$

Magnetic field errors tolerances for booster calculated by the equations (9) and (10) are shown in Table 1. According to the results of dynamic calculations such increase of beam emittance is totally acceptable.

Table 1. Magnetic field random errors tolerances.

\begin{tabular}{|c|c|c|}
\hline Resonance & $|\boldsymbol{\delta}|$ & Tolerance \\
\hline $3 \mathrm{Q}_{\mathrm{x}}=14$ & 0.4 & $32\left\langle\Delta B_{z} / B\right\rangle_{1, M}^{2}+\langle\Delta G / G\rangle_{n}^{2}<4.2 \cdot 10^{-5}$ \\
\hline $\mathrm{Q}_{\mathrm{x}}-\mathrm{Q}_{\mathrm{z}}=0$ & 0.05 & $40\left\langle\Delta B_{x} / B\right\rangle_{1, M}^{2}+\left\langle\alpha_{s}\right\rangle_{s}^{2}<8.9 \cdot 10^{-7}$ \\
\hline $\mathrm{Q}_{\mathrm{x}}-2 \mathrm{Q}_{\mathrm{z}}=-5$ & 0.1 & $\left\langle\Delta B_{x} / B\right\rangle_{2, \mu}^{2}+0,09\left\langle\Delta B_{x} / B\right\rangle_{2, n}^{2}<1.1 \cdot 10^{-5}$ \\
\hline $2 \mathrm{Q}_{\mathrm{x}}=10$ & 0.4 & $\left\langle\Delta B_{z} / B\right\rangle_{3, M}^{2}+0,01\left\langle\Delta B_{z} / B\right\rangle_{3, M}^{2}<1.2 \cdot 10^{-5}$ \\
\hline $2 \mathrm{Q}_{\mathrm{z}}=10$ & 0.3 & $\left\langle\Delta B_{x} / B\right\rangle_{3, M}^{2}+0,05\left\langle\Delta B_{x} / B\right\rangle_{3, M}^{2}<5.6 \cdot 10^{-5}$ \\
\hline
\end{tabular}

For example, for the first resonance $3 \mathrm{Q}_{x}=14\left\langle\Delta \mathrm{B}_{\mathrm{z}} / \mathrm{B}\right\rangle=\langle\Delta \mathrm{G} / \mathrm{G}\rangle=5 * 10^{-4}$ (as given in requirements specification). The inequality is $8 * 10^{-6}<4.2 * 10^{-5}$. So, these tolerances are satisfied the requirements and can be applicable to the assessment of magnetic field quality.

\section{Numerical simulation of magnetic field random errors}

Simulation of beam motion was carried out in MAD-X program over the period of $10^{3}$ turns. Dynamic aperture (DA) is defined as stable motion region after particle passing of given number of turns.

\subsection{Effective length spread for dipole magnets}

Effective length spread for dipole magnets bring about closed orbit deviation from the central position. Fig.2 presents a) the example of closed orbit deviation when $\sigma(B d L)=1 * 10^{-3}$; b) maximum orbit deviation to effective length spread diagram. Triple maximum value $(3 \sigma)$ was used for tolerance feasibility assessment on effective length spread. As it can be seen from Fig. $2 \mathrm{~b}$ the orbit deviation in horizontal direction doesn't exceed $9 \mathrm{~mm}$ in the case of $5 \cdot 10^{-4}$ relative spread.

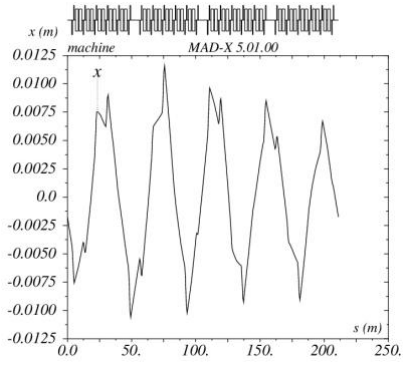

a)

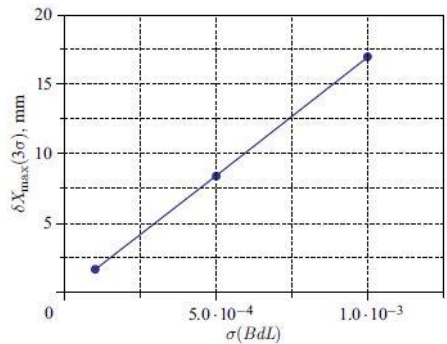

b)

Fig. 2. a) Closed orbit deviation along booster ring with $\sigma(B d L)=1 * 10^{-3}$ versus longitudinal coordinate; b) maximum orbit deviation $(3 \sigma)$ by 100 statistical samplings of effective length spread $\sigma(B d L)$.

\subsection{Effective length spread for quadrupole magnets}

Effective length spread for quadrupole magnets can cause excitation of parametric resonance and appropriate particle loss of accelerating beam. This effect can be estimated by DA attenuation. Figure 3 below provides the stable motion region on transverse phase planes with different values of effective length spread. 8 sextupoles were used for chromaticity correction ( 2 sextupoles for one quadrant). Gradients of magnetic field are $\mathrm{S}_{\mathrm{f}}: k_{2}=4.2 \cdot 25=105 \mathrm{~T} / \mathrm{m}^{2}, \mathrm{~S}$ : $k_{2}=-3.1 \cdot 25=-77.5 \mathrm{~T} / \mathrm{m}^{2}$. As can be seen from Fig.3, stable motion region is fully preserved if the relative spread is less than $5 \cdot 10^{-4}$. 


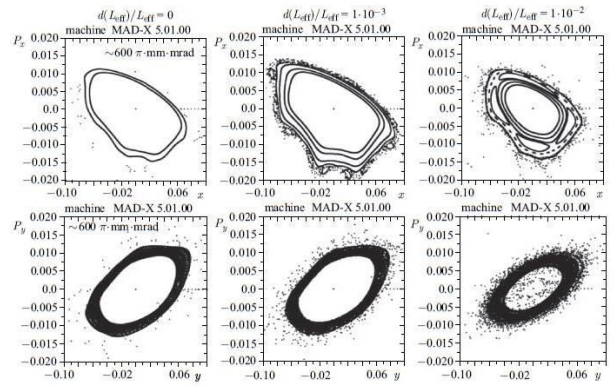

Fig. 3. Stable motion regions with different values of effective length spread in quadrupole magnets.

\subsection{Transverse displacements of quadrupole magnets around longitudinal axis}

Such displacements affect on generation of vertical and horizontal dipole components on ideal closed orbit line and its adequate distortion. This effect can be estimated by decrease of accelerator DA. Figure 4 a) presents the results of maximum allowed displacements simulation. Calculations were made similarly to 3.1 . As can be seen from this figure maximum disturbance of the closed orbit doesn't exceed $7 \mathrm{~mm}$.

\subsection{Rotation around longitudinal axis for dipole magnets}

Rotation around longitudinal axis results in horizontal component generation of magnetic field in accelerator median plane that in turn causes vertical orbital perturbation. The results of closed orbital perturbation versus the mean-square spread of rotation angle for all dipole magnets in the ring are shown on the Fig. 4 b). Maximum orbit deviation doesn't exceed $11 \mathrm{~mm}$ in our case.

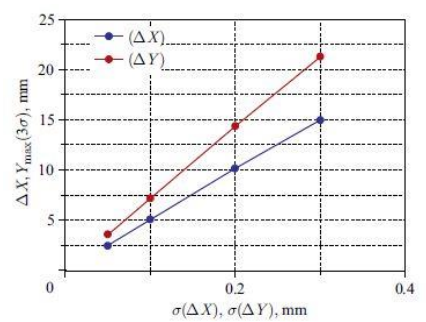

a)

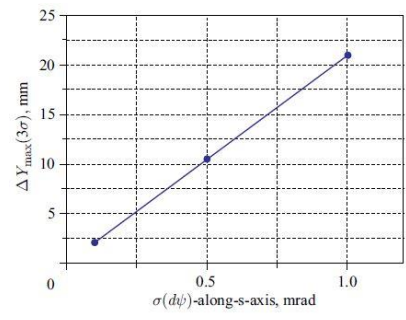

b)

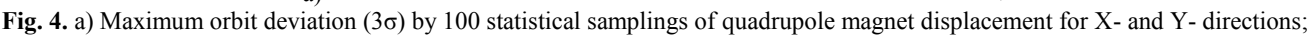
b) Maximum orbit deviation $(3 \sigma)$ by 100 statistical samplings of rotation angle for dipole magnets.

\subsection{Rotation around longitudinal axis for quadrupole magnets}

This effect results in connection between movements in X-and Y-directions. Such situation can reinforce the action on the coupled resonance bunch. Booster operating point $(4.8,4.85)$ is placed sufficiently far from coupled resonances. As can be seen from Fig. 5 stable motion region on phase planes with 5 mrad rotation angle doesn't change in comparison with the ideal layout under sextupoles for chromaticity correction.

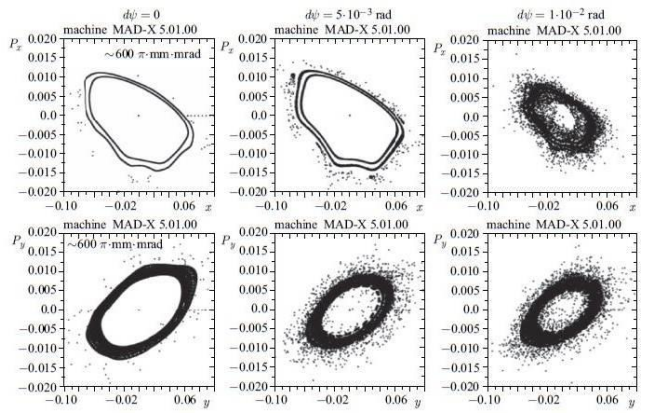

Fig. 5. Stable motion regions with different values of mean-square rotation angle in quadrupole magnets. 
On the ground of the received results, tolerances on the effective length spread and adjustment errors for booster unit are formulated in Table 2 .

Table 2. Tolerances on the effective length spread and adjustment errors.

\begin{tabular}{|c|c|c|}
\hline Error & Dipole magnet & Quadrupole magnet \\
\hline Relative effective length spread & $5 \cdot 10^{-4}$ & $5 \cdot 10^{-4}$ \\
\hline Transverse displacement around longitudinal axis, mm & - & \pm 0.1 \\
\hline Rotation around longitudinal axis, rad & $5 \cdot 10^{-4}$ & $10^{-3}$ \\
\hline
\end{tabular}

\subsection{Booster closed orbit and dynamic aperture under main influencing factors}

The results of booster closed orbit simulation and dynamic aperture are shown below (Fig.6 a) and b) respectively). They include the following errors of magnetic field and adjustment:

1. Effective length spread for dipole magnets $\sigma(B d L)=5 \cdot 10^{-4}$;

2. Rotation around longitudinal axis for dipole magnets $\sigma(d \varphi)=5 \cdot 10^{-4} \mathrm{rad}$;

3. Transverse displacement of quadrupole magnets $\sigma(\Delta X)=\sigma(\Delta Y)=0.1 \mathrm{~mm}$;

4. Effective length spread for quadrupole magnets $\sigma(G d L)=5 \cdot 10^{-4}$;

5. Rotation around longitudinal axis for quadrupole magnets $\sigma(d \varphi)=10^{-3} \mathrm{rad}$.
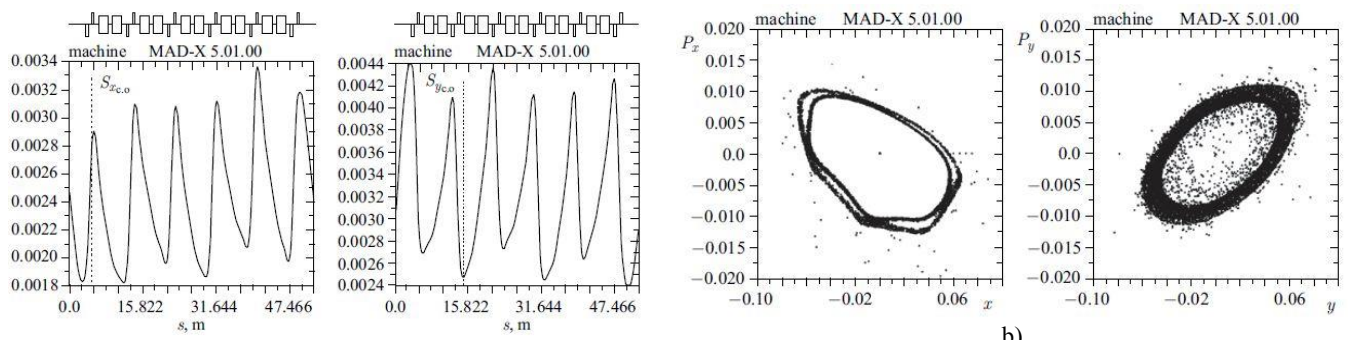

a)

b)

Fig. 6. a) Mean-square value of closed orbit deviation $(1 \sigma)$; b) Stable motion regions on the transverse phase.

For more details, please, see [6].

\section{Conclusions}

As the result of the research in linear and nonlinear dynamics of ion beam 197Au31+ in booster we have investigated the influence of random errors and adjustment errors on the closed orbit distortion and the betatron tune amplitude. Based on the results, tolerances about the quality of magnetic field and about alignment accuracy for booster units have been formulated. Maximum orbit deviation doesn't exceed $10 \mathrm{~mm}$ by both transverse directions. Analytical estimation and numerical simulation are conformed with each other.

\section{References}

1. MAD - Methodical Accelerator Design // CERN - BE/ABP Accelerator Beam Physics Group http://mad.web.cern.ch/mad/

2. P.L.Gluckstern, Distribution of the maximum orbit distortion for random distributed misalignment. PA, 8 , p.203-209 (1978).

3. I.N.Meshkov et al., Technical project of the NICA accelerator complex. Vol.2 (2015).

4. G.A.Guignard, General Treatment of Resonances in Accelerators. CERN-78-11, p.72 (1978).

5. V.I.Balbekov, P.N.Chirkov, The Octupole Correction System. IHEP 84-167 (1984).

6. A.V.Butenko et al., Magnetic field errors tolerances of Nuclotron booster. JINR P9-2017-18 (2017). 